\title{
Guillain-Barré and Acute Transverse Myelitis Overlap Syndrome Following Obstetric Surgery
}

\author{
Adina Stoian ${ }^{1,2}$, Anca Motataianu'2,3*, Zoltan Bajko 2,3 , Adrian Balasa ${ }^{4,5}$ \\ 1 Department of Pathophysiology, George Emil Palade University of Medicine, Pharmacy, Science, and Technology of \\ Targu Mures, Romania \\ 2 Neurology 1 Clinic, Emergency Clinical County Hospital of Targu Mures, Romania \\ ${ }^{3}$ Department of Neurology, George Emil Palade University of Medicine, Pharmacy, Science, and Technology of Targu \\ Mures, Romania \\ ${ }^{4}$ Neurosurgery Clinic, Emergency Clinical County Hospital of Targu Mures, Romania \\ ${ }^{5}$ Department of Neurosurgery, George Emil Palade University of Medicine, Pharmacy, Science, and Technology of \\ Targu Mures, Romania
}

\begin{abstract}
Introduction: There are rare reports of the occurrence of acute transverse myelitis and Guillain-Barré syndrome after various surgical procedures and general/epidural anaesthesia. The concomitant occurrence of these pathologies is very rare and is called Guillain-Barré and acute transverse myelitis overlap syndrome. In this article, we present the case of a second trimester pregnant patient who developed Guillain-Barré and acute transverse myelitis overlap syndrome. Case presentation: We report the case of a 16-year-old female patient who underwent a therapeutic termination of pregnancy two weeks prior to the onset of the disease with gradual development of a motor deficit with walking and sensitivity disorders, fecal incontinence. The diagnosis was based on clinical exam, electroneurography and spinal magnetic resonance imaging. Endocrinopathies, infectious diseases, autoimmune and inflammatory diseases, neoplastic diseases and vitamin deficiencies were ruled out. Our patient attended five sessions of therapeutic plasma exchange, followed by steroid treatment, intravenous immunoglobulin with minimum recovery of the motor deficit in the upper limbs, but without significant evolution of the motor deficit in the lower limbs. The patient was discharged on maintenance corticotherapy and immunosuppressive treatment with azathioprine. Conclusions: We report a very rare association between Guillain-Barré syndrome and acute transverse myelitis triggered by a surgical intervention with general anaesthesia. The overlap of Guillain-Barré syndrome and acute transverse myelitis makes the prognosis for recovery worse, and further studies are needed to establish the first-line therapy in these cases.
\end{abstract}

Keywords: Guillain-Barré syndrome, acute transverse myelitis, obstetric surgery, tetraparesis, intravenous immunoglobulins, therapeutic plasma exchange

Received: 17 January 2019 / Accepted: 30 January 2020

\section{INTRODUCTION}

The neurological complications that occur after surgery and epidural anaesthesia are usually considered to be local complications (epidural haematomas, abscesses, etc.) and are reported in the literature to have a low prevalence of $0.005-0.07 \%$ [1]. However, there are rare reports of the occurrence of acute transverse myelitis (ATM) and Guillain-Barré syndrome (GBS) after various surgical procedures and general/epidural anaesthesia [1]. The common subtypes of GBS are acute inflammatory demyelinating polyradiculoneu- ropathy (AIDP), which is the most frequent, and acute motor axonal neuropathy (AMAN), acute motor-sensory axonal neuropathy (AMSAN) and Miller-Fisher syndrome, the last of which is a less common variant of GBS $[1,2,3]$. ATM is an inflammatory process that occurs in the spinal cord; it usually affects multiple segments and is a medical emergency $[1,4]$. The concomitant occurrence of these pathologies is called GuillainBarré (GB) and ATM overlap syndrome [5]. In this article, we present the case of a patient who developed GB and ATM overlap syndrome. 


\section{CASE PRESENTATION}

We report the case of a 16-year-old female patient who underwent a therapeutic termination of pregnancy under general anaesthesia at 18 weeks of pregnancy for a malformed foetus (myelomeningocele) two weeks prior to the onset of the disease. She had no suggestive history of recent infections (respiratory or digestive) or vaccination in the preceding year. The first symptoms were paraesthesia that appeared in the lower limbs with ascending evolution towards the upper limbs and gradual development of a motor deficit with walking disorders. Two days after the first symptoms, the patient presented pain in the lower cervical and upper dorsal regions followed by worsening of the motor deficit in the lower limbs, acute urine retention and difficult bowel transit with fecal incontinence.

The neurological evaluation of muscle strength, assessed by Medical Research Council (MRC) grading, revealed general muscular hypotonia with flaccid tetraparesis grade $2 / 5$ in the upper limbs and grade $0 / 5$ in the lower limbs, global abolition of the deep tendon reflexes, superficial thermoalgesic anaesthesia at D1 level, apallesthesia of the lower limbs, acute urinary retention, and intestinal transit disorders. The suspicion of acute polyradiculoneuritis and ATM was raised.

Electroneurography (ENG) was performed and revealed axonal loss in all motor nerves (the amplitude of compound muscle action potential [CMAP] was reduced more than $70 \%$ ), with proximal conduction

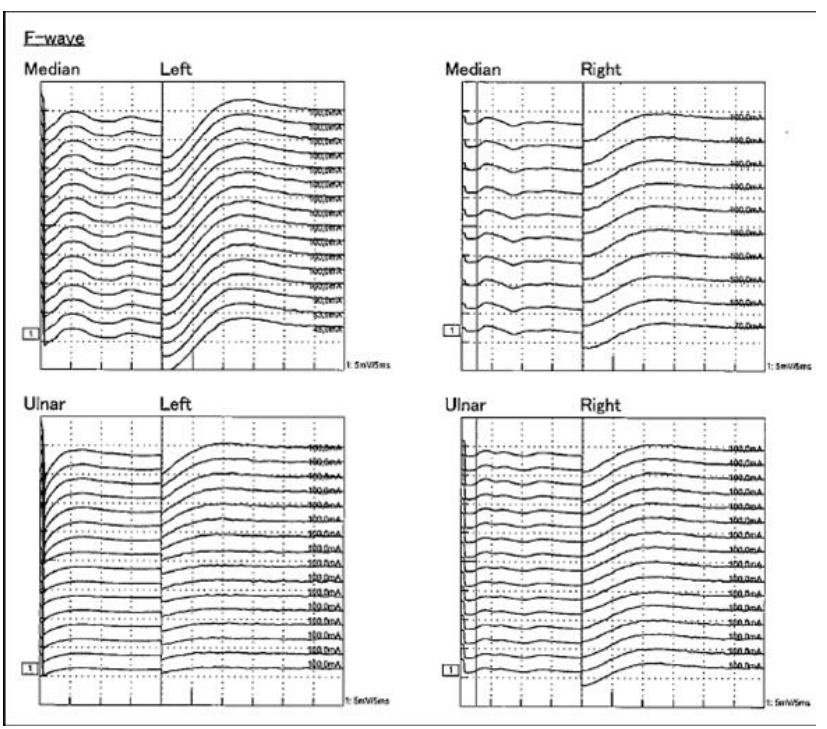

Fig. 1. Electroneurography revealed proximal conduction blocks detected by the absence of $F$ waves on both median and ulnar nerves bilaterally. blocks detected by the absence of $\mathrm{F}$ waves and normal sensory conduction (Figure 1 and 2).

The ENG aspect described above is characteristic for acute axonal motor polyradiculoneuritis (i.e. AMAN). Spinal magnetic resonance imaging (MRI) with intravenous gadolinium contrast medium revealed a T2 hyperintense lesion without enhancement (the patient was already receiving corticosteroid therapy) (Figure 3 ) and no specific signs for a neoplastic process. The cerebral MRI examination was within normal limits.

The corticospinal fluid examination did not reveal either abnormalities or bacterial, fungal or Mycobacterium tuberculosis infection. Laboratory examinations were extremely extensive and ruled out the presence of endocrinopathies, infectious diseases (hepatitis B and C, human immunodeficiency virus [HIV], syphilis, Epstein-Barr virus [EBV], cytomegalovirus [CMV], rubella, toxoplasmosis, human T-cell lymphotropic virus type 1 [HTLV-1], and Lyme disease serology), and autoimmune diseases (neuromyelitis optica [NMO] antibodies (aquaporin 4), anti-double stranded DNA [anti-dsDNA] antibodies, antinuclear antibodies
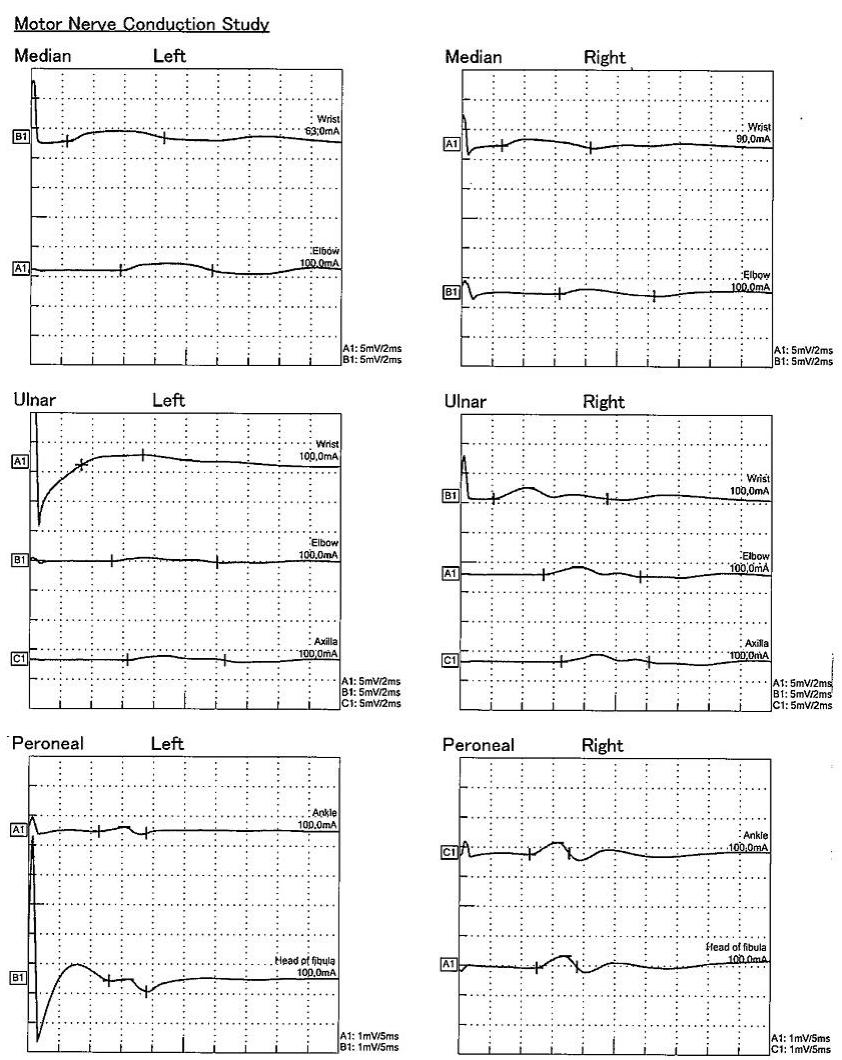

Fig. 2. Electroneurography was performed and revealed axonal loss in all motor nerves (the amplitude of compound muscle action potential [CMAP] was reduced more than $70 \%)$. 


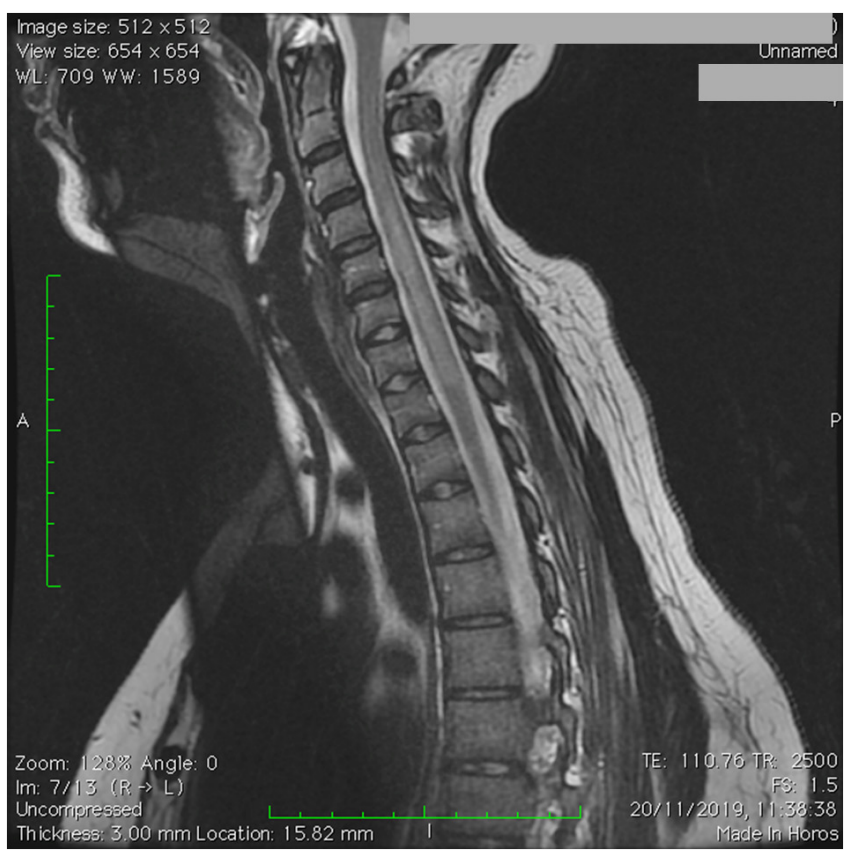

Fig. 3. Sagittal T2 weighted cervical MRI revealing a hyperintense longitudinally extensive spinal cord lesion

[ANAs], anti-neutrophil cytoplasmic antibodies [ANCAs], complement fractions, erythrocyte sedimentation rate [ESR], and antiphospholipid antibodies were within normal limits). Serology for Campylobacter jejuni and Mycoplasma pneumoniae was not available in our case. Vitamin B12 deficiency was excluded, serum protein electrophoresis revealed no anomalies, and the level of angiotensinconvertase was normal. The patient underwent a thoracoabdominal-pelvic computed tomography scan that was within normal limits, ruling out paraneoplastic myelitis.

Our patient attended five sessions of therapeutic plasma exchange with 1-1.5 plasma volumes changed every session, followed by intravenous pulse steroid therapy of $1 \mathrm{gram} /$ day of methylprednisolone for five consecutive days, and then 0.4 gram/kilogram of intravenous immunoglobulin (IVIg) daily for another five consecutive days, with minimum recovery of the motor deficit in the upper limbs, but without significant evolution of the motor deficit in the lower limbs.

Control cervical MRI examination described mild worsening and a slight extension of the lesions to T2 level (Figure 4).

After ten days from the last dose of steroid therapy another intravenous pulse steroid therapy was restarted for five consecutive days; this was followed by minor regression of the motor deficit in the lower limbs. The patient was discharged on maintenance corticotherapy and immunosuppressive treatment with azathioprine.

\section{DISCUSSION}

An April 2019 review identified 23 cases of GBS and ATM overlap [5]; after this review doctors from Pakistan published details of another case in August 2019 [6]. Similar to our case, most of the 24 cases were re-

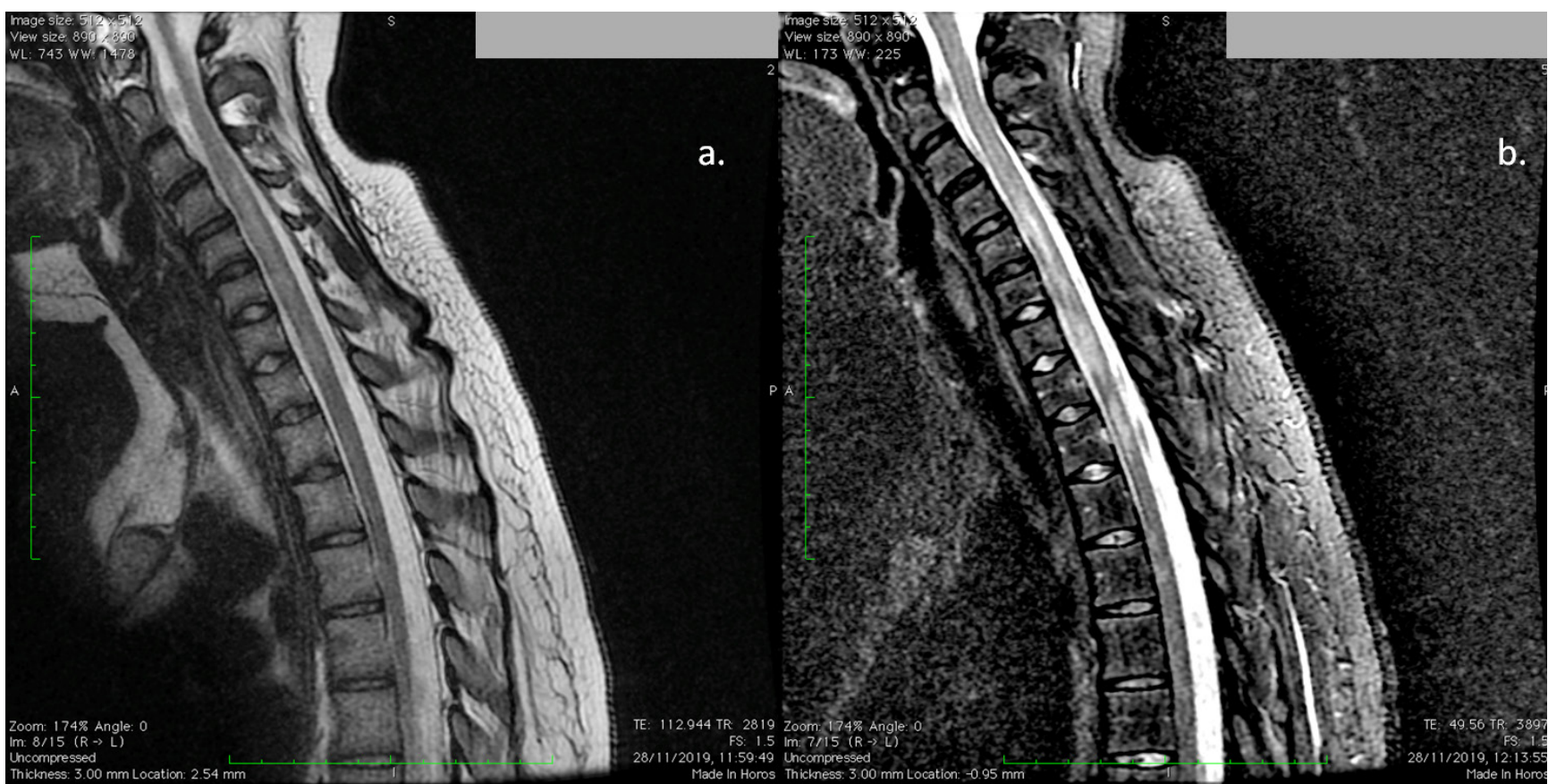

Fig. 4. Sagittal a. T2 weighted; b. Short tau inversion recovery (STIR) cervical MRI revealing a hyperintense longitudinally extensive spinal cord lesion 
ported in the paediatric population $[5,6]$. Most of the cases were preceded by different gastrointestinal or respiratory tract infections [6]. There was a predominance of females among the cases and a very young mean age (21.3 years) which suggest an autoimmune substrate that can be triggered by infections or vaccines [5]. We consider the trigger factor for our 16-year-old female patient to be surgery/general anaesthesia. In $91.3 \%$ of the cases in the aforementioned review, infection or vaccine administration was detected as the triggering factor [5]. Our paraclinical investigations did not show the existence of an acute infection; the patient had not received vaccines in the preceding year.

In the early stages of the disease, the diagnosis may be delayed in the absence of a neurologist in the case management team. Because the signs and symptoms of GBS may be superimposed on those of ATM, with high localization affecting the cervical spine, a correct diagnosis is very important for the patient to receive the best and most appropriate treatment. Nerve conduction studies and spinal cord MRI are also important tools for diagnosis.

There are currently no guidelines to establish the first-choice therapy for GBS and ATM overlap $[5,6]$. Therapeutic plasma exchange, intravenous IVIg and corticosteroid therapy are known to be effective for ATM $[2,3]$, but corticosteroid therapy has not been shown to improve recovery from GBS [7]. Both therapeutic plasma exchange and IVIg are recommended as a first-line therapy for GBS $[6,8]$. In accordance with clinical guidelines for GBS and ATM, our patient attended five sessions of therapeutic plasma exchange with 1-1.5 plasma volumes changed per session, without clinical improvement, followed by intravenous pulse steroid therapy of 1 gram/day of methylprednisolone for five consecutive days, and then 0.4 gram/kilogram of IVIg daily for another five consecutive days. After IVIg administration, the motor deficit of the upper limbs regressed from grade $0 / 5 \mathrm{MRC}$ to grade $3 / 5$ MRC proximally and grade 2/5 MRC distally, but the motor deficit of the lower limbs remained unchanged, suggesting favourable resolution of GBS after IVIg but not of transverse myelitis, which was not changed clinically and showed mild worsening and a slight extension to T2 level on MRI examination. The patient was transferred from the paediatric intensive care unit in the neurology clinic, and intravenous pulse steroid therapy was restarted for five consecutive days with slow taper- ing of methylprednisolone during the hospitalisation period (a total of 15 grams of methylprednisolone during a period of 40 days). After high doses of corticosteroids, a slight improvement of the motor deficit of the lower limbs was noted, from grade $0 / 5 \mathrm{MRC}$ to grade 2/5 MRC at discharge. Urinary retention and intestinal transit remained unchanged, and at discharge the patient received maintenance treatment with corticosteroids and immunosuppression with azathioprine. The patient was scheduled for another hospital admission after one month to perform clinical, neurophysiological and MRI reassessment.

Wolf et al. showed that if corticosteroid therapy and therapeutic plasma exchange have no effect in severe cases of ATM, IVIg and immunosuppressive therapy can be administered, which is in line with therapeutic approach used for our patient $[5,9]$. The logical assumption is that IVIg administration (which is efficient for GBS) $[5,10]$ combined with corticosteroid therapy (which is efficient for ATM) $[5,11]$ may have a beneficial effect on GB and ATM overlap syndrome, but the percentage of those responding appropriately is not very high (46.2\%) [5]. This was seen also in our patient; although she showed regression of the motor deficit in the upper limbs, at the time of discharge, she still had severe motor deficit in the lower limbs and significant sphincter disorders due to the inadequate therapeutic response of myelitis. In the review mentioned above, three patients received combined therapy with plasmapheresis, IVIg and corticosteroids followed by cyclophosphamide, and all of them showed only partial recovery, as in the case of our patient [5].

The concurrent occurrence of GBS and ATM as an overlap syndrome is rarely reported in the literature. The overlap may be explained by the existence of a common myelin epitope in the peripheral nervous system (PNS) and the central nervous system (CNS) that is subjected to immune-mediated self-aggression [6, 12]. Cases of GBS triggered by surgery have previously been reported to occur in the first eight weeks after the procedure, especially in patients with a history of neoplastic disease (gastrointestinal carcinoma, blood and skin cancers, prostate cancer, or gynaecological cancer) or a positive history of other autoimmune diseases (Sjögren syndrome, giant-cell arteritis, antiphospholipid antibody syndrome, ulcerative colitis, type 1 diabetes mellitus, sarcoidosis, Graves' disease, or rheumatoid arthritis) [13]. Some authors have suggested that post- 
surgical GBS is the result of nerve root exposure during surgery, with nerve antigen release that triggers an antigen-mediated autoimmune response directed against epitopes that are present in the PNS; we may assume that the CNS is also affected by the same mechanism $[13,14]$. Another presumed mechanism is the possible interaction of lipid-soluble pharmacological agents used in general anaesthesia with the myelin of the PNS, and by implication with that of the CNS, and thereby initiating the immunological process $[13,15]$. Similarly, we found some reports of ATM, but no reports of GB and ATM overlap syndrome, being triggered by surgery or general/epidural anaesthesia $[1,16]$.

Patients with the AMSAN subtype of GBS have a poorer prognosis than those with the AIDP subtype, and usually do not have the ability to move independently six months after the onset of the disease $[5,17]$. Campylobacter jejuni and Mycoplasma pneumoniae infections are reported to be associated with GBS but serology for these infections was not available in our case $[18,19]$. According to Hughes et al., the AMAN subtype of GBS is associated with rapid evolution towards disability, and recovery is a slow and long process $[5,18]$. In addition, the combination of ATM and GBS, especially the axonal form (AMSAN or AMAN) of the latter, is associated with poorer recovery compared to the occurrence of only one of these demyelinated disorders, which is confirmed by the evolution of the overlap syndrome in our patient $[5,20]$. Four cases of ATM and GB (with the AMAN phenotype) overlap syndrome have been reported in Gou and Zhang's (2019) review [5]; our case is the fifth possible identified with this pathology, and all reported cases had a poor outcome in the short term.

\section{CONCLUSIONS}

We report a very rare association between GBS and ATM which, to our knowledge, is the first case reported in the literature to be triggered by a surgical intervention with general anaesthesia. The overlap of GBS and ATM makes the prognosis for recovery worse, and further studies are needed to establish the first-line therapy in these cases.

\section{INFORMED CONSENT}

Informed consent forms were signed by the patient's parents, and they agreed to our use of their daughter's medical records for publication.

\section{CONFLICT OF INTEREST}

None to declare.

\section{REFERENCES}

1. Shimada T, Yufune S, Tanaka M, Akai R, Satoh Y, Kazama T. Acute transverse myelitis arising after combined general and thoracic epidural anesthesia. JA Clin Rep. 2015;1(1):1-4

2. Leonhard SE, Mandarakas MR, Gondim FAA, Bateman K, Ferreira MLB, Cornblath DR, van Doorn PA, Dourado ME, Hughes RAC, Islam B, Kusunoki S, Pardo CA, Reisin R, Sejvar JJ, Shahrizaila N, Soares C, Umapathi T, Wang Y, Yiu EM, Willison $\mathrm{HJ}$, Jacobs BC. Diagnosis and management of Guillain-Barré syndrome in ten steps. Nat Rev Neurol. 2019;15(11):671-683

3. Choudary JR, Alladi S, Mridula KR, Oddu DB, Rao MV, Hemanth C, Clinical Outcome of Guillain-Barre Syndrome with various treatment methods and cost effectiveness: A Study from tertiary care centre in South India. Neurology Asia, 2014;19(3):263-270

4. Bălaşa R, Bajkó Z, Moţăţăianu A, Maier A, Maier S. Neuromyelitis optica associated with painful paroxysmal dystonia: case report and literature review. Acta Neurol Belg. 2015;115(2):169-171

5. Guo F, Zhang YB. Clinical features and prognosis of patients with Guillain-Barré and acute transverse myelitis overlap syndrome.Clin Neurol Neurosurg. 2019;181:127-132

6. Javed Z, Malik WT, Lodhi OUH. A Rare Case of Acute Transverse Myelitis (ATM) and Acute Motor and Sensory Axonal Neuropathy (AMSAN) Overlap. Cureus. 2019;11(8):e5426

7. Hughes RA, Brassington R, Gunn AA, van Doorn PA., Corticosteroids for Guillain-Barré syndrome. Cochrane Database Syst Rev., 2016;10:CD001446

8. Hughes RA, Cornblath DR. Guillain-Barré syndrome. Lancet. 2005;366:1653-1666.

9. Wolf $\mathrm{VL}$, Lupo PJ, Lotze TE. Pediatric acute transverse myelitis overview and differential diagnosis. J Child Neurol. 2012;27(11):1426-36

10. Martens-Le Bouar $\mathrm{H}$, Korinthenberg R. Polyradiculoneuritis with myelitis: a rare differential diagnosis of Guillain-Barré syndrome. Neuropediatrics. 2002;33(2):93-6

11. Bălaşa R, Maier S, Bajko Z, Motataianu A, Crişan A, Bălaşa A. Longitudinally extensive transverse myelitis in neuromyelitis optica: a prospective study of 13 Caucasian patients and literature review. Acta Neurol Belg. 2015;115(4):635-642

12. Topcu Y, Bayram E, Karaoglu P, Yis U, Guleryuz H, Kurul SH. Coexistence of myositis, transverse myelitis, and Guillain Barré syndrome following mycoplasma pneumoniae infection in an adolescent. J Pediatr Neurosci. 2013;8:59-63

13. Nagarajan E, Rubin M, Wijdicks EFM, Hocker SE.GuillainBarré syndrome after surgical procedures. Neurology: Clinical Practice. 2017;7:1-9 
Available online at: www.jccm.ro

14. Gensicke H, Datta AN, Dill P, Schindler C, Fischer D. Increased incidence of Guillain-Barré syndrome after surgery. Eur J Neurol 2012;19:1239-1244

15. Korn-Lubetzki I, Abramsky O. Acute and chronic demyelinating inflammatory polyradiculoneuropathy: association with autoimmune diseases and lymphocyte response to human neuritogenic protein. Arch Neurol 1986;43:604-608

16. Shimada T, Yufune S, Tanaka M, Akai R, Satoh Y, Kazama T. Acute transverse myelitis arising after combined general and thoracic epidural anesthesia. JA Clin Rep. 2015;1(1):1-6
The Journal of Critical Care Medicine 2020;6(1) • 79

17. Winer JB. Guillain-Barré syndrome: clinical variants and their pathogenesis .J Neuroimmunol. 2011;231(1-2):70-72

18. Hughes RA, Cornblath DR. Guillain-Barre syndrome. Lancet, 2005;366(9497):1653-1666

19. Theodorou D, Sutton R, Ram D, G McCullagh. G386(P) Think twice: concurrent acute transverse myelitis and guillain-barre syndrome. Archives of Disease in Childhood 2017;102:A152

20. Howell KB, Wanigasinghe J, Leventer RJ, Ryan MM. Concomitant transverse myelitis and acute motor axonal neuropathy in an adolescent. Pediatr Neurol. 2007;37(5):378-381 